\title{
Launch Vehicle Production and Operations Cost Metrics
}

\author{
Michael D. Watson ${ }^{1}$, \\ NASA Marshall Space Flight Center, Huntsville, AL 35812 \\ and \\ James R. Neeley ${ }^{2}$ and Ruby F. Blackburn ${ }^{3}$ \\ Jacobs ESSSA Group, Huntsville, AL 35806
}

\begin{abstract}
Traditionally, launch vehicle cost has been evaluated based on $\$ / \mathrm{Kg}$ to orbit. This metric is calculated based on assumptions not typically met by a specific mission. These assumptions include the specified orbit whether Low Earth Orbit (LEO), Geostationary Earth Orbit (GEO), or both. The metric also assumes the payload utilizes the full lift mass of the launch vehicle, which is rarely true even with secondary payloads. ${ }^{1,2,3}$ Other approaches for cost metrics have been evaluated including unit cost of the launch vehicle and an approach to consider the full program production and operations costs. ${ }^{4}$ Unit cost considers the variable cost of the vehicle and the definition of variable costs are discussed. The full program production and operation costs include both the variable costs and the manufacturing base. This metric also distinguishes operations costs from production costs, including pre-flight operational testing. Operations costs also consider the costs of flight operations, including control center operation and maintenance. Each of these 3 cost metrics show different sensitivities to various aspects of launch vehicle cost drivers. The comparison of these metrics provides the strengths and weaknesses of each yielding an assessment useful for cost metric selection for launch vehicle programs.
\end{abstract}

\section{Cost Model}

There are several approaches that have been used to structure and analyze launch vehicle costs. ${ }^{5,6}$ Work Breakdown Structures (WBS) and Product Breakdown Structures (PBS) provide structures from which to analyze cost. Each of the structures provides a different view of the cost. The WBS provides a labor centric view, allowing management of the workforce in developing, manufacturing, and operating the launch vehicle. This provides an organizational centric view on the cost but can be difficult to identify the unit cost of the launch vehicle as labor categories often cut across multiple vehicle elements or components. A PBS provides the product centric view, allowing specific cost of each component, assembly, and element of the launch vehicle to be tracked. This unit costs fall directly out of the PBS and allow specific cost drivers of the vehicle to be identified. The PBS does not clearly indicate cross cutting support functions where one labor unit may support multiple components, assemblies, or elements.

The costs for a launch vehicle are often divided between development and production and operations (P\&O). The WBS between the development phase of the program and the P\&O phase can be very different. During development, engineering disciplines are the focus, and often the WBS is centered on these discipline functions. During $\mathrm{P} \& \mathrm{O}$, production costs are a much stronger driver and the WBS is aligned more to the production disciplines and the operations disciplines. The PBS also differs between the development and the P\&O phase. Development costs are driven by design, analysis, integration, and test costs. These costs collectively define the cost of specific components, assemblies, and elements within the launch vehicle. For the P\&O phase, the PBS is driven mainly by the production costs. Operations costs are typically smaller than the cost of producing the launch vehicle.

Production costs are the primary driver to $\mathrm{P} \& \mathrm{O}$ phase costs. Production costs are all costs associated with the manufacture of the launch vehicle elements as represented by element unit cost. These include materials, production labor, factory test and inspection, and integration performed at the manufacturing sites. Operations costs are all costs associated with the operation of the launch vehicle after completion of manufacturing. These include green run

\footnotetext{
${ }^{1}$ System Engineer, System Engineering Management Office, Chief Engineers Office

${ }^{2}$ Logistics, Engineering and Science Services Skills Augmentation

${ }^{3}$ Cost Analyst, Engineering and Science Services Skills Augmentation
} 
testing associated with each flight, transportation, launch site assembly, flight operations, post flight analysis, and sustaining engineering.

Life Cycle Costs (LCC) combines the development costs and the P\&O costs into a single number. This provides an indication of total program costs over the full life of the vehicle. There are several difficulties with this approach. The drivers for development costs and $\mathrm{P} \& \mathrm{O}$ costs are quite different as mentioned above. In addition, the life cycle costs must assume a program duration. These durations are difficult to define and often are set based on return on investment (profitability) time required to recuperate the development costs. For government funded launch vehicles, the development costs are generally funded as a package. P\&O costs are funded on a periodic basis (following the countries funding cycle which is annually in the United States). The Space Shuttle, for example, was intended as a 10 year life cycle which ended up running 30 years. Thus, P\&O costs, in context of the LCC were 3 times greater than originally planned. Duration, then, is not as important as the annual cost to produce and operate the launch vehicle. Thus, LCC, has not generally been a good measure of the launch vehicle costs as the duration is difficult to define.

Cost models build on these products to produce various cost estimates and metrics. Many cost estimating models exist, and, similar to the estimating approaches and methodologies, no single cost model can be used for all purposes. ${ }^{7}$ Some models are a basic construct to be used as a tool while other models are estimating environments that can be all-inclusive and automate many functions for the cost estimator. A model can also use a variety of estimating methodologies and direct inputs to complete a full estimate. For each methodology, there are a multitude of both commercially available and government developed or owned models. Cost modeling features that are useful for space vehicles include automation of applying inflation, learning, time-phasing, what-if analysis, risk analysis, WBS, iterative PBS estimating, bottoms-up engineering builds, and reporting.

\section{Cost Drivers}

There are several factors which drive the cost of launch vehicles. ${ }^{8}$ These include development testing, manufacturing base, learning curve, launch site operations, and inflation. Most of these are different between the two phases. Inflation is common to development and $\mathrm{P} \& \mathrm{O}$ phases.

Costs in the development phase are driven strongly by test costs. Hardware testing of full scale testing is very expensive involving the production cost of the test unit and the cost of the test facility integration, operation, and possible refurbishment. Similarly, software testing in an integrated hardware laboratory also drives costs. In this case, the cost of the flight like avionics and validated simulation models are key drivers. Other cost drivers include transportation system development for full scale stages, ground support equipment at the launch site, launch site development, and any manufacturing capability development.

$\mathrm{P} \& \mathrm{O}$ costs are driven by production costs. In particular the overhead rates with maintaining a manufacturing base can be a significant portion of costs. The cost of maintenance of equipment and facilities, training and retention of the workforce, and retained viability during any low launch periods can take a significant portion of

program costs. Manufacturing processes also drive costs, mainly through labor required to operate and maintain the equipment. Material costs are not generally substantial when compared with the overall unit costs. There is a learning curve associated with production activities that leads to a reduction in cost over the first few years of a program. ${ }^{1,2}$ Learning curve reductions results from lessons learned leading to improved efficiencies in the manufacturing process. This reduction only occurs during the P\&O phase and is somewhat dependent on the manufacturing approaches chosen and the organizational structure. Operations costs at the launch site also drive costs. These costs include the maintenance of servicing facilities, launch pad services, launch towers, consumables (i.e., fuel and oxidizer) and the control center. These can account for $20-35 \%$ of the annual launch vehicle program costs. ${ }^{9}$

Inflation rates are important in both development and $\mathrm{P} \& \mathrm{O}$ phases and must be accounted for in the cost models. The annual compounding of inflation on a large development cost or $\mathrm{P} \& \mathrm{O}$ cost can add significant cost to the program over time.

\section{Cost Metrics}

As illustrated in Figure 1, three options have been evaluated to estimate and monitor launch vehicle costs taking the above factors into consideration. The first method is to separately compare the development costs and the P\&O costs directly with the budget. As discussed in cost drivers, the drivers for these two development phases differ. Thus separate cost models should be used in the management of these two phases. This requires the use of a PBS for the development phase to calculate the development costs (testing, transportation, GSE, manufacturing preparation). This is a fairly common practice in launch vehicle development. 
A similar, yet separate, approach should be taken for the P\&O phase. Specific launch vehicle costs (labor and materials) should be calculated to produce the launch vehicle unit cost. This cost will be a constant, regardless of the actual mass of the payload suite being launched or the orbit to be achieved. The manufacturing base should be estimated separately as these costs are independent of actual unit production. The concept of manufacturing base absorbing some of the unit production costs will be discussed below.

Operations costs are generally defined as post manufacturing costs. Specific preflight testing (such as green run testing of engines or stages) is considered post manufacturing activities and therefore part of these operational costs. Launch site facility costs, vehicle assembly costs, launch costs are all part of the operations costs. Similar to the manufacturing base, launch site facility costs are independent of the unit operations costs. Vehicle assembly and launch costs are part of the unit operations costs.

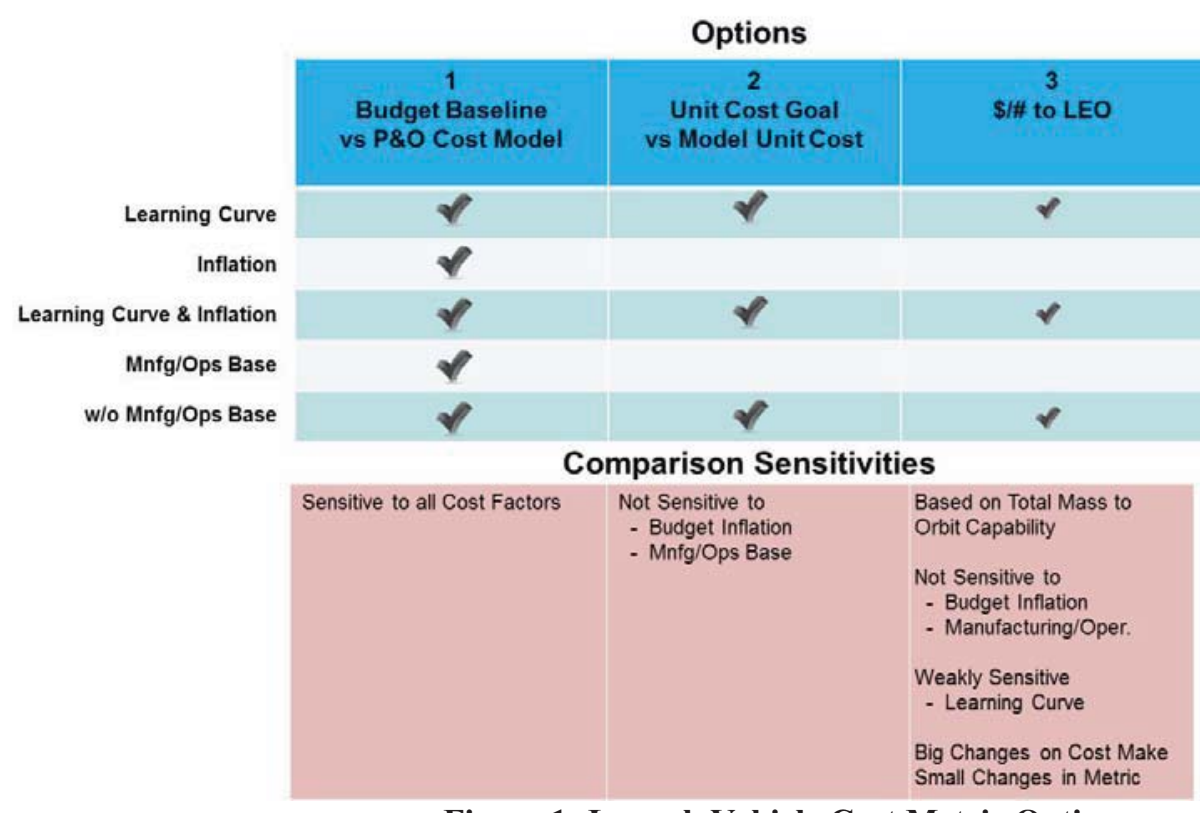

Figure 1: Launch Vehicle Cost Metric Options

Looking at the $\mathrm{P} \& \mathrm{O}$ costs further, a cost metric which explicitly and clearly captures all the factors discussed above provides the most sensitive measure to the various cost drivers. Manufacturing base and operational base costs are often amortized over the launch rate. This gives a misleading view that these costs decrease with this rate. They are actually constant or expand if the launch rate is much greater or much lower. If the launch rate grows significantly, exceeding manufacturing base capacity, then the base must be expanded to meet the increased flight rate. This expansion increases the cost of the manufacturing and operational bases. Conversely, when the flight rate is low, or substantially reduced below the manufacturing base and operational base through put, costs tend to increase as well. In this case cost increases occur due to lower efficiencies of the production lines operating below designed through put and increased maintenance on idle equipment where idle systems experience freeze up, lose calibration, experience increased corrosion, and soft goods expire over time. Since the systems are idle, failures due to these cases are not often detected until manufacturing and operations restart. If capacity is leased out, the leased uses affect machine wear and life. Low utilization of work force tends to lead to many continuous improvement ideas for production and operation performance. This leads to increased cost to upgrade or modify systems that are not necessarily inefficient. The Space Shuttle costs provide an example of this effect. ${ }^{10}$ So amortizing manufacturing base and operational base costs over the flight rate does not provide a realistic view of the cost trends. Treating these as an annual cost provides a clear view of their portion of the total program cost. As these can be a larger percentage of the actual costs.

Annual manufacturing process and operations costs are clearly seen in this metric. The learning curve affects the manufacturing process costs and the unit operations costs for the first few years or units of the program. This is clearly seen in the annual $P \& O$ costs for the launch vehicle.

Inflation also affects the $\mathrm{P} \& \mathrm{O}$ costs annually. The program must be able to plan for inflationary effects. This is especially important if a flat cost profile is desired. This will require continuous cost reduction efforts throughout 
the life of the launch vehicle program. Viewing the inflationary effects in the annual costs allows a more clear understanding of the effectiveness of the cost reduction efforts and management of the overall cost profile.

As mentioned above, the manufacturing base and the operational facility costs can absorb some of the cost of a launch vehicle. This occurs when some of the base is maintenance of necessary skills to produce and operate the launch vehicle. The amount of this varies with manufacturing site and launch site. Figure 2 illustrates this effect. As shown in the figure, the ideal model is to account for the portions contributing to the unit cost as part of the unit cost. The unit cost must be understood first, in order to determine the cost overlap. This ultimately makes the manufacturing base smaller as long as at least one vehicle is in production. If there is a total lapse in production, due to an accident investigation and recovery for example, then the expansion of the base to maintain critical skills should be accounted as the cost of the production stop and restart costs.

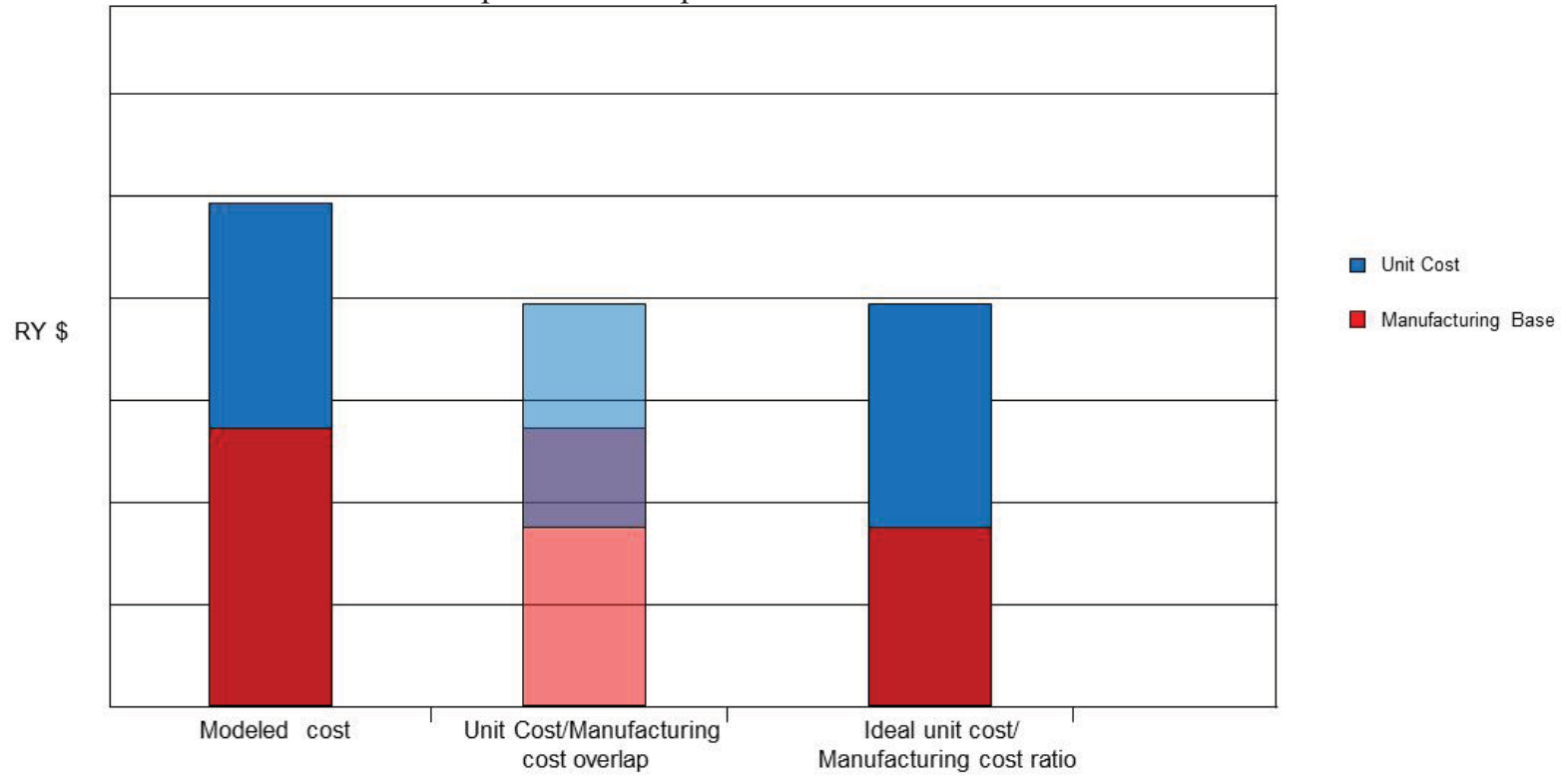

Figure 2: Unit Cost and Manufacturing Base Overlap

The second option is to generate a goal for the unit cost and compare to the modeled unit cost. Unit cost is an important measure of the launch vehicle cost and incorporates all of the production costs drivers described above. Learning curve reduction, as a production artifact, is reflected in the unit cost and causes the unit cost to be a variable rather than a set cost over the first few units produced. This must be understood and planned for when using unit cost metrics. If only the first unit cost is used as metric, then the metric will not show the effects of the learning curve reductions. Unit operations costs, green run testing, launch vehicle assembly, and launch, should be included in the unit cost metric. Production unit cost combined with operation unit costs provides a total cost for the launch vehicle service.

The unit cost metric, however, masks some details of the total launch vehicle program costs and is less sensitive to certain cost drivers. Manufacturing base and operations facilities costs are not clearly visible in the unit cost. These can be amortized into the calculated unit cost but require an assumed flight rate. If the actual flight rate is different, particularly if much lower, then the metric does not accurately depict the launch vehicle cost. The Space Shuttle, for example, had early estimates of $50-150$ flights per year ${ }^{11}$ but only averaged 5 flights per year over the life of the program. 10

Inflation effects are also not represented in unit cost metrics unless the metric varies with the inflation rate and elapsed time from the base year unit cost. Similar to learning curve reductions, inflation causes the unit cost metric to be variable and not set. This makes the metric difficult to use. If only the base year is used as a metric, the effects of inflation are not seen in the metric.

The third option is the traditional cost/mass to orbit. This approach has not proven to be a reliable predictor of actual costs. Cost $/$ mass $(\$ / \mathrm{lbm}$ or $€ / \mathrm{Kg}$ ) has to assume both an orbit (which affects payload mass) and a maximum payload mass. Rarely do launch vehicles carry their exact maximum mass to a single reference orbit. The orbit generally varies with the primary payload requirements. Thus the costs are generally higher for a given launch than the metric would lead you to understand. 
A separate aspect of mass based metrics is to estimate development costs based on launch vehicle mass. This is also an inexact relationship. As the vehicle is made lighter; the manufacturing processes become more complex and expensive. As a result, the vehicle costs go up with decreasing mass. This was shown in early cost estimates of launch vehicle configuration options for the Space Launch System. There is a direct mass to cost relationship if the manufacturing processes are exactly the same. Then the heavier vehicle will cost more (mainly through the increased materials, which are small cost changes). As soon as manufacturing processes must be made more complex or detailed, the relationship inverts and the vehicle requiring the increased manufacturing complexity becomes more expensive, even though often times with lower vehicle mass.

This cost/mass to orbit metric essentially a unit cost/payload mass metric. Thus, it has the same insensitivities to cost drivers as the unit cost metric does. Manufacturing base and operational facility costs are not seen clearly in the metric. Amortizing these costs only complicates the metric adding flight rate as a dependent variable to payload mass and orbit location. Learning curve and inflation is not seen in this metric unless the metric is treated as variable. Dividing unit cost by mass essentially scales down the effects of cost changes by 4 or 5 orders of magnitude. Thus large changes in costs have small effect in this metric.

The number of assumptions required to employ the cost/mass to orbit metric generate a large uncertainty for this metric. Variation in actual payload mass launched, orbit to be achieved, and flight rate can leave the metric very non-descriptive of the launch vehicle actual P\&O costs for a specific mission. This was seen in the early estimates of the Space Shuttle versus the actual costs. ${ }^{12,13}$

\section{Summary}

Several factors in cost modeling and cost metrics have been considered for launch vehicles. Launch vehicle costs drivers are different for the development phase and the P\&O phase. During the development phase testing, launch site development, transportation and GSE development, and manufacturing base development are major cost drivers. During the $\mathrm{P} \& O$ phase, manufacturing base, operational base, production processes (labor), operations, and green run testing are major cost drivers. Learning curve reductions can also be significant early in the $\mathrm{P} \& \mathrm{O}$ phase. Inflation is a factor across both phases. The effects of all of these cost drivers must be visible in the cost metrics. The traditional unit cost/payload mass to orbit does not show good sensitivity to learning curve, inflation, or the manufacturing base. The division by payload mass significantly reduces the magnitude of cost changes in the metric making large changes have small effects. This metric has multiple dependencies including flight rate, actual payload mass per launch, and mission orbit to achieve. These create large uncertainties in the metric results. Unit cost alone does not have the reduction in magnitude changes and is not dependent on payload mass or orbit. So it has better sensitivity to production costs such as learning curve effects (if the metric is variable by launch). However, unit cost still is not sensitive to inflation, manufacturing base, and operational base costs. Amortizing the manufacturing and operational bases requires an additional dependency on flight rate which diminishes the utility of the metric. Using the annual development cost, and separately, the annual $\mathrm{P} \& \mathrm{O}$ costs provides a cost metric that is sensitive to all launch vehicle cost drivers. This does not create a fixed metric, but one that varies as the cost drivers vary. Cost effects scale directly such that large cost impacts make commensurate cost shifts in the metric. Managing cost against these metric provides the most effective means to view cost changes.

\section{References}

${ }^{1}$ Hermann, T. M.; Akin, D. L.; “A critical parameter Optimization of Launch Vehicle Costs”, AIAA Space 2005, 2005-6680, pp. 1-13.

${ }^{2}$ Pempie, P.; Lefur, T.; "Considerations on Launch Vehicle Cost Modeling”, $54^{\text {th }}$ International Astronautical

Congress, Bremen, Germany, 2003, IAC-03-V.2.08, pp 1- 6.

${ }^{3}$ Whitehead, J. C.; "Launch Vehicle Cost: A Low Tech Analysis", $36^{\text {th }}$ AIAA Joint Propulsion Conference, Huntsville, Alabama, 2000, AIAA 2000-3140, pp. 1-10.

${ }^{4}$ Prince, A.; Apgar, H.; "Constellation Program Affordability”, A!AA Space 2007, Long Beach, California, AIAA 2007-6006, pp. 1-11.

${ }^{5}$ Shishko, R.; "Modeling Operations Costs for Human Exploration Architectures”, AIAA Space 2013, 2013-5489, pp. $1-13$.

${ }^{6}$ Bieler, T.; Abitzsch, S.; "Cost Management” in Handbook of Space Technology, ed. By Ley, Wilfried ; Wittmann, Klaus ; Hallmann, Willi, AIAA, Virginia, 2009, pp 793-808.

${ }^{7}$ NASA Cost Analysis Division; "2008 NASA Cost Estimating Handbook", pp. 1-33. 
${ }^{8}$ Hauer, S.; Tabata, G.; Waters, R. ; “Launch Vehicle Cost Analysis and System Evaluation”, AIAA Heterogeneous Combustion Conference, 10.2514/6.1963-1410, 1963, pp. 51 - 56.

${ }^{9}$ Braun, M. T.; Groo, R. M.; Cohn, B.; "Launch Operations of Minimum Cost Design Launch Vehicles", AIAA Launch Operations Meeting, Cocoa Beach, Florida, 1970, AIAA 70-240, pp. 1-6.

${ }^{10}$ Rutledge, W.; "Launch Vehicle Cost Trends and Potential for Cost Containment", AIAA Space Programs and Technologies Conference, Huntsville, Alabama, 1993, pp. 1-9.

${ }^{11}$ Mosley, W.; "Tomorrow - Low Cost Launch Operations", AIAA Launch Operations Meeting, Cocoa Beach , Florida, 1970, AIAA 70-242, pg. 2.

${ }^{12}$ NASA; "Shuttle Cost Per Flight FY1993 Budget", June 25, 1992.

13 "FY91 Budget to OMB Shuttle Cost Per Flight", NSTS Program, October 6, 1989. 